\title{
Integral constraints in multiple-scales problems
}

\author{
S. J. CHAPMAN and S. E. MCBURNIE \\ Mathematical Institute, University of Oxford, ROQ, Woodstock Road, Oxford OX2 6GG, UK \\ emails: chapman@maths.ox.ac.uk,semcburnie@googlemail.com
}

(Received 11 November 2014; accepted 19 November 2014; first published online 13 January 2015)

\begin{abstract}
Asymptotic homogenisation via the method of multiple scales is considered for problems in which the microstructure comprises inclusions of one material embedded in a matrix formed from another. In particular, problems are considered in which the interface conditions include a global balance law in the form of an integral constraint; this may be zero net charge on the inclusion, for example. It is shown that for such problems care must be taken in determining the precise location of the interface; a naive approach leads to an incorrect homogenised model. The method is applied to the problems of perfectly dielectric inclusions in an insulator, and acoustic wave propagation through a bubbly fluid in which the gas density is taken to be negligible.
\end{abstract}

Key words: homogenisation; multiple scales; perfect dielectric; rigid inclusions; bubbly fluid; effective medium; averaging; coarse graining

\section{Introduction and motivation}

The technique of asymptotic homogenisation via multiple scales is widely used for a variety of problems involving a (locally) periodic microstructure for which bulk or effective equations are required [5-7]. Typical applications include the derivation of Darcy flow from the Stokes equations, homogenisation of a rapidly varying porosity/conductivity/diffusion coefficient, effective elastic properties of composite materials, and the transmission of acoustic waves through bubbly fluids.

Our interest in this paper lies with problems for which the unit cell contains more than one region; for example, the unit cell may contain two materials in which the (perhaps different) field equations must be solved, separated by an interface. Such microstructures arise naturally and frequently; the examples of composite materials and bubbly liquids above fall into this category. If the conditions on the interface between these regions are natural, representing for example continuity of flux, and the field equations are the same on both sides of the interface, then such interfaces pose no additional complication over and above the usual multiple scales procedure. In fact, if the interface arises simply through a discontinuous coefficient in the field equation (the Young's modulus for example), the multiple scales procedure can be carried out assuming a smoothly varying coefficient, and the sharp interface limit taken subsequently (the two limiting procedures commute).

However, if the interface conditions involve a global balance in the form of an integral constraint, then more care needs to be taken: we will see that a naive approach leads to an incorrect homogenised model. Such a constraint appears for example when considering 
an array of electrically conducting inclusions in an insulating matrix: the net charge on each inclusion must be zero. We give more examples in Section 3.

Our motivation for the present study came from a claim in [2] that the multiple scales limit did not commute with the limit of small gas density for the problem of acoustic waves in a bubbly fluid. We will show that in fact the limits do commute, but that a careful handling of the interface is necessary when the small gas density limit is taken first.

We note that the method of multiple scales is just one approach to deriving effectivemedium equations for problems with a detailed microstructure. A related technique is volume averaging [10], which often results in the same effective-medium equations [3]. An entirely different approach involves treating the microstructure as stochastic, and averaging not over space but over realisations of the microstructure [1,9]. It is usually very difficult to estimate the effective properties from such a description, since doing so requires an infinite set of statistical correlation functions, which are generally never known [8]. Nevertheless, variational methods can often be used to obtain upper and lower bounds on the effective material properties [4]. We are not aware of any attempt to apply either of these methods to problems involving integral constraints on microinclusions.

We first present a one-dimensional paradigm problem which enables us to illustrate the failings of the naive multiple scales approach, as well as the way to correct it. We then present in Section 3 a number of physical examples in which integral constraints arise. In Section 4, we use one of these examples to illustrate the application of the method in three dimensions. In Section 5, we return to the problem of acoustic wave propagation through a bubbly fluid. Finally, in Section 6, we present our conclusions.

\section{A one-dimensional paradigm problem}

We consider the following one-dimensional paradigm problem:

$$
\frac{\mathrm{d}}{\mathrm{d} x}\left(a\left(\frac{x}{\epsilon}\right) \frac{\mathrm{d} y}{\mathrm{~d} x}\right)=f\left(\frac{x}{\epsilon}\right)
$$

where $a(X)$ is assumed 1-periodic in $X$ and is given by

$$
a(X)= \begin{cases}a_{1} \text { if } & 0 \leqslant X<\frac{1}{3} \\ a_{2} \text { if } & \frac{1}{3}<X<\frac{2}{3} \\ a_{1} \text { if } & \frac{2}{3}<X<1\end{cases}
$$

and the source term $f(X)$ is assumed 1-periodic in $X$ and is given by

$$
f(X)= \begin{cases}f_{1} \text { if } & 0 \leqslant X<\frac{1}{3} \\ f_{2} \text { if } & \frac{1}{3}<X<\frac{2}{3} \\ f_{1} \text { if } & \frac{2}{3}<X<1\end{cases}
$$


For ease of exposition, we assume that the $f_{i}$ and $a_{i}(i=1,2)$ are constant, though the modification if this is not the case is easy to make. At the interfaces $x / \epsilon \bmod 1=1 / 3,2 / 3$ both $y$ and $a \mathrm{~d} y / \mathrm{d} x$ should be continuous.

Introducing the fast scale $X=x / \epsilon$, writing $y=y(x, X)$ with $y$ 1-periodic in $X$ and treating $x$ and $X$ as independent as is usual in multiple scales gives

$$
\left(\epsilon \frac{\partial}{\partial x}+\frac{\partial}{\partial X}\right)\left(a(X)\left(\epsilon \frac{\partial y}{\partial x}+\frac{\partial y}{\partial X}\right)\right)=\epsilon^{2} f(X) .
$$

Expanding $y=y_{0}+\epsilon y_{1}+\cdots$ gives at leading order

$$
\frac{\partial}{\partial X}\left(a(X)\left(\frac{\partial y_{0}}{\partial X}\right)\right)=0
$$

for which the relevant solution is $y_{0}=y_{0}(x)$. At next order in (2.4) we find

$$
\frac{\partial}{\partial X}\left(a(X)\left(\frac{\mathrm{d} y_{0}}{\mathrm{~d} x}+\frac{\partial y_{1}}{\partial X}\right)\right)=0,
$$

so that

$$
\frac{\mathrm{d} y_{0}}{\mathrm{~d} x}+\frac{\partial y_{1}}{\partial X}=\frac{Q(x)}{a(X)}
$$

say. Integrating over $X$ and using the periodicity of $y_{1}$ gives

$$
\frac{\mathrm{d} y_{0}}{\mathrm{~d} x}=Q(x) \int_{0}^{1} \frac{\mathrm{d} X}{a(X)} .
$$

At next order in (2.4), we find

$$
\frac{\partial}{\partial x}\left(a(X)\left(\frac{\mathrm{d} y_{0}}{\mathrm{~d} x}+\frac{\partial y_{1}}{\partial X}\right)\right)+\frac{\partial}{\partial X}\left(a(X)\left(\frac{\partial y_{1}}{\partial x}+\frac{\partial y_{2}}{\partial X}\right)\right)=f(X),
$$

i.e.

$$
\frac{\mathrm{d} Q}{\mathrm{~d} x}+\frac{\partial}{\partial X}\left(a(X)\left(\frac{\partial y_{1}}{\partial x}+\frac{\partial y_{2}}{\partial X}\right)\right)=f(X),
$$

Integrating over $X$ (and using the continuity of $\left.a(X)\left(\partial y_{1} / \partial x+\partial y_{2} / \partial X\right)\right)$ gives

$$
\frac{\mathrm{d} Q}{\mathrm{~d} x}=\int_{0}^{1} f(X) \mathrm{d} X .
$$

Thus the leading-order homogenised problem is

$$
\frac{\mathrm{d}}{\mathrm{d} x}\left(\frac{1}{\int_{0}^{1} a(X)^{-1} \mathrm{~d} X} \frac{\mathrm{d} y_{0}}{\mathrm{~d} x}\right)=\int_{0}^{1} f(X) \mathrm{d} X .
$$

For our particular case of $a$ and $f$ being step functions this reduces to

$$
\frac{3 a_{2} a_{1}}{2 a_{2}+a_{1}} \frac{\mathrm{d}^{2} y_{0}}{\mathrm{~d} x^{2}}=\frac{2 f_{1}+f_{2}}{3} .
$$


In the limit $a_{2} \rightarrow \infty,(2.5)$ becomes

$$
\frac{3 a_{1}}{2} \frac{\mathrm{d}^{2} y_{0}}{\mathrm{~d} x^{2}}=\frac{2 f_{1}+f_{2}}{3}
$$

Our aim is to show that this result can be obtained by first passing to the limit $a_{2} \rightarrow \infty$, and then using a multiple-scales expansion, though care needs to be taken.

\subsection{The limit $a_{2} \rightarrow \infty$}

Taking $a_{2} \rightarrow \infty$ in (2.1) gives

$$
\begin{array}{rlrl}
a_{1} \frac{\mathrm{d}^{2} y}{\mathrm{~d} x^{2}} & =f_{1} & \text { for } & \frac{x}{\epsilon}(\bmod 1) \in(0,1 / 3) \cup(2 / 3,1), \\
\frac{\mathrm{d}^{2} y}{\mathrm{~d} x^{2}}=0 & \text { for } \quad & \frac{x}{\epsilon}(\bmod 1) \in(1 / 3,2 / 3),
\end{array}
$$

with $y$ continuous at $x / \epsilon \bmod 1=1 / 3,2 / 3$ and

$$
\frac{\mathrm{d} y}{\mathrm{~d} x}=0 \quad \text { at } \frac{x}{\epsilon}(\bmod 1)=\frac{1}{3}^{+} \text {and } \frac{2}{3}^{-} .
$$

It is clear immediately that this limit problem is not well-posed, and an additional condition needs to be given. Equations (2.8) and (2.9) give that in each middle third of the periodic cell $y$ is constant, and continuity of $y$ then links the values of $y$ at $x / \epsilon(\bmod 1)=1 / 3^{-}$ and $2 / 3^{+}$. However, we have no information on the derivative at these points, and this is the extra information which must be supplied. We expect this information to depend on $f_{2}$, since this constant appears in the limiting homogenised problem (2.6), but does not appear in (2.7)-(2.9). To derive this extra condition, we integrate (2.1) over $x$ from $x=\epsilon n+\epsilon / 3$ to $x=\epsilon n+2 \epsilon / 3$ for some $n \in \mathbb{Z}$ to give

$$
a_{2}\left[\frac{\mathrm{d} y}{\mathrm{~d} x}\right]_{\epsilon(n+1 / 3)^{+}}^{\epsilon(n+2 / 3)^{-}}=\int_{\epsilon(n+1 / 3)}^{\epsilon(n+2 / 3)} \frac{\mathrm{d}}{\mathrm{d} x}\left(a_{2} \frac{\mathrm{d} y}{\mathrm{~d} x}\right) \mathrm{d} x=\int_{\epsilon(n+1 / 3)}^{\epsilon(n+2 / 3)} f_{2} \mathrm{~d} x=\frac{\epsilon}{3} f_{2} .
$$

We can then use the continuity of flux to give

$$
\left.a_{1} \frac{\mathrm{d} y}{\mathrm{~d} x}\right|_{\epsilon(n+2 / 3)^{+}}-\left.a_{1} \frac{\mathrm{d} y}{\mathrm{~d} x}\right|_{\epsilon(n+1 / 3)^{-}}=\frac{\epsilon}{3} f_{2} .
$$

This condition is retained in the limit $a_{2} \rightarrow \infty$, and is the extra piece of information we need to make (2.7)-(2.9) well-posed.

Now the equation $y=$ constant for $x / \epsilon(\bmod 1) \in(1 / 3,2 / 3)$ is not suitable for multiple scales (the constant may be different in different cells). To perform a multiple-scales analysis it is crucial to write instead

$$
\frac{\mathrm{d} y}{\mathrm{~d} x}=0 \quad \text { when } \frac{1}{3}<\frac{x}{\epsilon} \bmod 1<\frac{2}{3} .
$$


Converting (2.7) and (2.11) into multiple scales form, introducing $X$ as before, we have

$$
\begin{array}{cc}
a_{1}\left(\epsilon \frac{\partial}{\partial x}+\frac{\partial}{\partial X}\right)^{2} y=\epsilon^{2} f_{1} \quad \text { for } X \in(0,1 / 3) \cup(2 / 3,1), \\
\left(\epsilon \frac{\partial}{\partial x}+\frac{\partial}{\partial X}\right) y=0 \quad \text { for } X \in(1 / 3,2 / 3),
\end{array}
$$

with $y$ continuous at $X=1 / 3,2 / 3$. We expand write $y=y(x, X)$ with $y$ 1-periodic in $X$ and expand

$$
y=y_{0}(x, X)+\epsilon y_{1}(x, X)+\cdots .
$$

As before we find $y_{0}=y_{0}(x)$. At $O(\epsilon)$ we find

$$
\begin{aligned}
a_{1} \frac{\partial}{\partial X}\left(\frac{\partial y_{1}}{\partial X}+\frac{\mathrm{d} y_{0}}{\mathrm{~d} x}\right) & =0 & & \text { for } X \in(0,1 / 3) \cup(2 / 3,1), \\
\left(\frac{\partial y_{1}}{\partial X}+\frac{\mathrm{d} y_{0}}{\mathrm{~d} x}\right) & =0 & & \text { for } X \in(1 / 3,2 / 3) .
\end{aligned}
$$

Thus

$$
a_{1}\left(\frac{\partial y_{1}}{\partial X}+\frac{\mathrm{d} y_{0}}{\mathrm{~d} x}\right)= \begin{cases}Q_{L}(x) & \text { for } X \in(0,1 / 3), \\ Q_{R}(x) & \text { for } X \in(2 / 3,1),\end{cases}
$$

say. Now by periodicity and by continuity of $y$ at $X=1 / 3,2 / 3$, we have

$$
0=\int_{0}^{1} \frac{\partial y_{1}}{\partial X} \mathrm{~d} X=\frac{1}{3}\left(\frac{Q_{L}}{a_{1}}-\frac{\mathrm{d} y_{0}}{\mathrm{~d} x}\right)+\frac{1}{3}\left(\frac{Q_{R}}{a_{1}}-\frac{\mathrm{d} y_{0}}{\mathrm{~d} x}\right)-\frac{1}{3} \frac{\mathrm{d} y_{0}}{\mathrm{~d} x},
$$

so that

$$
\frac{\mathrm{d} y_{0}}{\mathrm{~d} x}=\frac{\left(Q_{L}+Q_{R}\right)}{3 a_{1}}
$$

At $O\left(\epsilon^{2}\right)$, equation (2.12) gives

$$
\begin{array}{ll}
a_{1} \frac{\partial}{\partial X}\left(\frac{\partial y_{2}}{\partial X}+\frac{\partial y_{1}}{\partial x}\right)+\frac{\mathrm{d} Q_{L}}{\mathrm{~d} x}=f_{1} & \text { for } X \in(0,1 / 3), \\
a_{1} \frac{\partial}{\partial X}\left(\frac{\partial y_{2}}{\partial X}+\frac{\partial y_{1}}{\partial x}\right)+\frac{\mathrm{d} Q_{R}}{\mathrm{~d} x}=f_{1} & \text { for } X \in(2 / 3,1) .
\end{array}
$$

Integrating (2.19) and (2.20) and using periodicity gives

$$
\begin{aligned}
\frac{2}{3} f_{1}-\frac{1}{3}\left(\frac{\mathrm{d} Q_{L}}{\mathrm{~d} x}+\frac{\mathrm{d} Q_{R}}{\mathrm{~d} x}\right) & =\int_{0}^{\frac{1}{3}^{-}}\left(f_{1}-\frac{\mathrm{d} Q_{L}}{\mathrm{~d} x}\right) \mathrm{d} X+\int_{\frac{2}{3}^{+}}^{1}\left(f_{1}-\frac{\mathrm{d} Q_{R}}{\mathrm{~d} x}\right) \mathrm{d} X \\
& =\left.a_{1}\left(\frac{\partial y_{2}}{\partial X}+\frac{\partial y_{1}}{\partial x}\right)\right|_{\frac{1}{3}^{-}}-\left.a_{1}\left(\frac{\partial y_{2}}{\partial X}+\frac{\partial y_{1}}{\partial x}\right)\right|_{\frac{2}{3}^{+}}
\end{aligned}
$$

To evaluate the right-hand side, we turn to our extra flux condition (2.10). It seems natural that in multiple scales form this equation should become

$$
\left.a_{1}\left(\epsilon \frac{\partial y}{\partial x}+\frac{\partial y}{\partial X}\right)\right|_{X=\frac{2}{3}^{+}}-\left.a_{1}\left(\epsilon \frac{\partial y}{\partial x}+\frac{\partial y}{\partial X}\right)\right|_{X=\frac{1}{3}^{-}}=\frac{\epsilon^{2}}{3} f_{2} .
$$


If this were true then at $O(\epsilon)$ it implies $Q_{L}=Q_{R}$, while at $O\left(\epsilon^{2}\right)$ it gives

$$
\left.a_{1}\left(\frac{\partial y_{1}}{\partial x}+\frac{\partial y_{2}}{\partial X}\right)\right|_{X=\frac{2}{3}^{+}}-\left.a_{1}\left(\frac{\partial y_{1}}{\partial x}+\frac{\partial y_{2}}{\partial X}\right)\right|_{X=\frac{1}{3}^{-}}=\frac{f_{2}}{3} \text {. }
$$

Substituting this into (2.21) gives

$$
\frac{2 f_{1}+f_{2}}{3}=\frac{1}{3}\left(\frac{\mathrm{d} Q_{L}}{\mathrm{~d} x}+\frac{\mathrm{d} Q_{R}}{\mathrm{~d} x}\right)
$$

which combines with (2.18) to give

$$
a_{1} \frac{\mathrm{d}^{2} y_{0}}{\mathrm{~d} x^{2}}=\frac{2 f_{1}+f_{2}}{3} .
$$

Comparing with (2.6), we see that the right-hand side is correct, but that the effective diffusivity is incorrect by a factor of $3 / 2$.

The mistake arose when we naively put the left-hand side of (2.22) into multiple scales form. We must be careful to take account of the $O(\epsilon)$ drift in $x$ between $X=1 / 3$ and $X=2 / 3$. For ease of notation we write

$$
Q=a_{1} \frac{\mathrm{d} y}{\mathrm{~d} x} .
$$

Given an arbitrary point $x$, the interior interfaces in the unit cell lie at $x_{L}=x-\epsilon b+\epsilon / 3$ and $x_{R}=x-\epsilon b+2 \epsilon / 3$, where $b=x / \epsilon-\lfloor x / \epsilon\rfloor$ is the phase shift between $x$ and the periodic structure. Then, equation (2.10) becomes

$$
Q\left(\left(x-\epsilon b+\frac{2 \epsilon}{3}\right)^{+}\right)-Q\left(\left(x-\epsilon b+\frac{\epsilon}{3}\right)^{-}\right)=\frac{\epsilon}{3} f_{2} .
$$

In multiple scales form this gives

$$
\begin{aligned}
\frac{\epsilon}{3} f_{2}=Q & \left(x+\epsilon\left(-b+\frac{2}{3}^{+}\right), \frac{2}{3}^{+}\right)-Q\left(x+\epsilon\left(-b+\frac{1}{3}^{-}\right), \frac{1}{3}^{-}\right) \\
=Q & \left(x, \frac{2}{3}^{+}\right)+\epsilon\left(-b+\frac{2}{3}\right) Q_{x}\left(x, \frac{2^{+}}{3}\right)+\cdots \\
& -Q\left(x, \frac{1^{-}}{3}\right)-\epsilon\left(-b+\frac{1}{3}\right) Q_{x}\left(x, \frac{1}{3}^{-}\right)+\cdots,
\end{aligned}
$$

At $O(1)$ equation $(2.27)$ gives

$$
Q_{0}\left(x, \frac{2}{3}^{+}\right)-Q_{0}\left(x, \frac{1}{3}^{-}\right)=0
$$

which, with (2.17), gives $Q_{L}(x)=Q_{R}(x)$. Then at $O(\epsilon)$ equation (2.27) gives

$$
Q_{1}\left(x, \frac{2}{3}^{+}\right)-Q_{1}\left(x, \frac{1}{3}^{-}\right)=\frac{f_{2}}{3}-\frac{1}{3} \frac{\mathrm{d} Q_{L}}{\mathrm{~d} x},
$$


which is

$$
\left.a_{1}\left(\frac{\partial y_{1}}{\partial x}+\frac{\partial y_{2}}{\partial X}\right)\right|_{X=\frac{2}{3}^{+}}-\left.a_{1}\left(\frac{\partial y_{1}}{\partial x}+\frac{\partial y_{2}}{\partial X}\right)\right|_{X=\frac{1}{3}^{-}}=\frac{f_{2}}{3}-\frac{1}{3} \frac{\mathrm{d} Q_{L}}{\mathrm{~d} x} .
$$

Note the extra term on the right-hand side by comparison with (2.23). This extra term is just what we need so that after substituting into (2.21) and using (2.18) we arrive at (2.6).

\subsection{Flux formulation}

In the analysis above the flux $Q$ arises as a natural variable. It is illustrative to perform the same multiple-scales procedure after recasting the equations using $Q$ as a dependent variable. We write the equations as

$$
Q=a \frac{\mathrm{d} y}{\mathrm{~d} x}, \quad \frac{\mathrm{d} Q}{\mathrm{~d} x}=f,
$$

with $y$ and $Q$ continuous at the interfaces $x / \epsilon \bmod 1=1 / 3,2 / 3$. In multiple scales form with our particular choice of $a$ and $f$ this becomes

$$
\begin{aligned}
Q_{X}+\epsilon Q_{x} & =\epsilon f_{1} & & \text { for } X \in(0,1 / 3) \cup(2 / 3,1), \\
Q_{X}+\epsilon Q_{x} & =\epsilon f_{2} & & \text { for } X \in(1 / 3,2 / 3), \\
a_{1} y_{X}+a_{1} \epsilon y_{x} & =\epsilon Q & & \text { for } X \in(0,1 / 3) \cup(2 / 3,1), \\
y_{X}+\epsilon y_{x} & =0 & & \text { for } X \in(1 / 3,2 / 3) .
\end{aligned}
$$

Note that in this case, since we have retained (2.32), we have not needed to impose an additional integral condition. We expand

$$
y=y_{0}(x, X)+\epsilon y_{1}(x, X)+\cdots, \quad Q=Q_{0}(x, X)+\epsilon Q_{1}(x, X)+\cdots,
$$

and assume that each term is 1-periodic in $X$. At leading order we find $\partial y_{0} / \partial X=$ $\partial Q_{0} / \partial X=0$, which, with continuity at $X=1 / 3$ and $X=2 / 3$, gives $y_{0}=y_{0}(x)$, $Q_{0}=Q_{0}(x)$. Equating $O(\epsilon)$ terms in (2.31)-(2.34) gives

$$
\begin{aligned}
\frac{\partial Q_{1}}{\partial X}+\frac{\partial Q_{0}}{\partial x} & =f_{1} & & \text { for } X \in(0,1 / 3) \cup(2 / 3,1), \\
\frac{\partial Q_{1}}{\partial X}+\frac{\partial Q_{0}}{\partial x} & =f_{2} & & \text { for } X \in(1 / 3,2 / 3), \\
a_{1} \frac{\partial y_{1}}{\partial X}+a_{1} \frac{\partial y_{0}}{\partial x} & =Q_{0} & & \text { for } X \in(0,1 / 3) \cup(2 / 3,1), \\
\frac{\partial y_{1}}{\partial X}+\frac{\partial y_{0}}{\partial x} & =0 & & \text { for } X \in(1 / 3,2 / 3) .
\end{aligned}
$$

Integrating (2.36) and (2.37) and using periodicity and continuity of $Q_{1}$ and $X=1 / 3$ and $X=2 / 3$ gives

$$
0=\int_{0}^{1} \frac{\partial Q_{1}}{\partial X} \mathrm{~d} X=\frac{2 f_{1}+f_{2}}{3}-\frac{\partial Q_{0}}{\partial x}
$$


Now integrating (2.38) and (2.39) and using periodicity and continuity of $y_{1}$ yields

$$
0=\int_{0}^{1} \frac{\partial y_{1}}{\partial X} \mathrm{~d} X=\frac{2}{3} \frac{Q_{0}}{a_{1}}-\frac{\mathrm{d} y_{0}}{\mathrm{~d} x}
$$

Eliminating $Q_{0}$ from (2.40) and (2.41) gives

$$
\frac{3 a_{1}}{2} \frac{\mathrm{d}^{2} y_{0}}{\mathrm{~d} x^{2}}=\frac{2 f_{1}+f_{2}}{3}
$$

as required.

Although the flux formulation works very well in one-dimension, it does not generalise easily to higher dimensions. In particular, in the bubble fluid problem which follows, it is not possible to use $\mathbf{u}$ and $p$ as variables and avoid integral constraints because of a lack of information on $\mathbf{u}$ inside the bubble. In one dimension, the flux $Q$ is independent of the fast scale to leading order; the same is not true in higher dimensions.

\section{Physical examples of problems involving integral constraints}

In addition to the propagation of acoustic waves through a bubbly fluid mentioned in the introduction, there are a number of other physical situations which lead to a spatially uniform field within an inclusion, whose value is determined by an integral constraint. In particular, we note the following examples.

\subsection{High contrast diffusion}

If a material $\Omega_{\mathrm{e}}$ with low thermal conductivity contains closed regions $\Omega_{\mathrm{i}}$ in which there is a much higher conductivity then the conductivity may be taken to be infinite in the inclusions, so that the temperature $T$ is constant there. In that case

$$
\begin{aligned}
\rho_{\mathrm{e}} c_{\mathrm{e}} \frac{\partial T}{\partial t} & =k_{\mathrm{e}} \nabla^{2} T & & \text { in } \Omega_{\mathrm{e}}, \\
{[T]_{\mathrm{i}}^{\mathrm{e}} } & =0, & & \\
T & =T_{\mathrm{i}}(t) & & \text { in each } \Omega_{\mathrm{i}},
\end{aligned}
$$

where $\rho, c$ and $k$ are density, specific heat and thermal conductivity respectively, and [· $]_{i}^{e}$ denotes the jump in the enclosed quantity across the interface. The temperature in each inclusion determined by the global energy balance

$$
\left|\Omega_{\mathrm{i}}\right| \rho_{\mathrm{i}} c_{\mathrm{i}} \frac{\mathrm{d} T_{\mathrm{i}}}{\mathrm{d} t}=\left.k_{\mathrm{e}} \int_{\partial \Omega_{\mathrm{i}}} \frac{\partial T}{\partial n}\right|_{\mathrm{e}} \mathrm{d} S .
$$




\subsection{Rigid inclusions in an elastic material}

If an elastic material $\Omega_{\mathrm{e}}$ contains rigid inclusions $\Omega_{\mathrm{i}}$, then in the dynamic case

$$
\begin{array}{rlrl}
\rho_{\mathrm{e}} \frac{\partial^{2} \mathbf{u}}{\partial t^{2}} & =\left(\lambda_{\mathrm{e}}+\mu_{\mathrm{e}}\right) \nabla(\nabla \cdot \mathbf{u})+\mu_{\mathrm{e}} \nabla^{2} \mathbf{u} & & \text { in } \Omega_{\mathrm{e}}, \\
{[\mathbf{u}]_{\mathrm{i}}^{\mathrm{e}}} & =0, & \\
\mathbf{u} & =\mathbf{U}_{\mathrm{i}}(t)+\mathbf{R}_{\mathrm{i}}(t) \wedge\left(\mathbf{x}-\mathbf{x}_{G_{\mathrm{i}}}\right) \quad & \text { in each } \Omega_{\mathrm{i}},
\end{array}
$$

where $\mathbf{x}_{G_{\mathrm{i}}}$ is the position of the centre of mass, $\rho$ is the density and $\lambda$ and $\mu$ are the Lamé constants. The displacement and rotation of each inclusion determined by force and torque balances. For example, the force balance is

$$
\left|\Omega_{\mathrm{i}}\right| \rho_{\mathrm{i}} \frac{\mathrm{d}^{2} \mathbf{u}_{\mathrm{i}}}{\mathrm{d} t^{2}}=\left.\int_{\partial \Omega_{\mathrm{i}}} \boldsymbol{\sigma} \cdot \mathbf{n}\right|_{\mathrm{e}} \mathrm{d} S,
$$

i.e.

$$
\left|\Omega_{\mathrm{i}}\right| \rho_{\mathrm{i}} \frac{\partial^{2} u_{\mathrm{i}, k}}{\partial t^{2}}=\left.\int_{\partial \Omega_{\mathrm{i}}}\left(\lambda_{\mathrm{e}} \frac{\partial u_{k}}{\partial x_{k}} n_{k}+\mu_{\mathrm{e}}\left(\frac{\partial u_{k}}{\partial x_{j}}+\frac{\partial u_{j}}{\partial x_{k}}\right) n_{j}\right)\right|_{\mathrm{e}} \mathrm{d} S,
$$

where the summation convention is applied.

\subsection{Electrically conducting inclusions in an insulator}

If an insulating material $\Omega_{\mathrm{e}}$ contains closed regions $\Omega_{\mathrm{i}}$ of conducting material then the electric field is zero in each inclusion, so that the electric potential $\phi$ is constant there. In that case

$$
\begin{aligned}
\nabla^{2} \phi & =0 & & \text { in } \Omega_{\mathrm{e}}, \\
{[\phi]_{\mathrm{i}}^{\mathrm{e}} } & =0, & & \\
\phi & =\phi_{\mathrm{i}}(t) & & \text { in each } \Omega_{\mathrm{i}} .
\end{aligned}
$$

The value in each inclusion determined by the condition of zero net charge

$$
\left.\int_{\partial \Omega_{\mathrm{i}}} \frac{\partial \phi}{\partial n}\right|_{\mathrm{e}} \mathrm{d} S=0
$$

\subsection{Perfectly dielectric inclusions}

Our final example is a material with perfectly dielectric inclusions. In general, the electric field in a dielectric material satisfies Poisson's equation

$$
\nabla \cdot(\varepsilon \nabla \phi)=-\rho,
$$

where $\phi$ is the electric potential, $\varepsilon$ is the permittivity, and $\rho$ is the charge density (which we suppose is given). If the material contains inclusions $\Omega_{\mathrm{i}}$ of permittivity $\varepsilon_{\mathrm{i}}$ embedded in 
a matrix $\Omega_{\mathrm{e}}$ of permittivity $\varepsilon_{\mathrm{e}}$ then at the boundary between the two regions

$$
\begin{aligned}
{[\phi]_{\mathrm{i}}^{\mathrm{e}} } & =0, \\
{\left[\varepsilon \frac{\partial \phi}{\partial n}\right]_{\mathrm{i}}^{\mathrm{e}} } & =0 .
\end{aligned}
$$

In the limit $\varepsilon_{\mathrm{i}} \rightarrow \infty$ the inclusions are perfectly dielectric and

$$
\begin{aligned}
\varepsilon_{\mathrm{e}} \nabla^{2} \phi & =-\rho & & \text { in } \Omega_{\mathrm{e}} \\
\nabla \phi & =0 & & \text { in } \Omega_{\mathrm{i}},
\end{aligned}
$$

with boundary condition

$$
[\phi]_{\mathrm{i}}^{\mathrm{e}}=0,
$$

Thus $\phi$ is constant on each inclusion (corresponding to zero electric field there as expected); however, the constant may be different on different inclusions. To close the problem, we need to add back the information which we have lost in taking the limit. Integrating (3.1) over $\Omega_{\mathrm{i}}$ and using (3.3) gives

$$
\left.\int_{\partial \Omega_{\mathrm{i}}} \varepsilon_{\mathrm{e}} \frac{\partial \phi}{\partial n}\right|_{\mathrm{e}} \mathrm{d} S=-\int_{\Omega_{\mathrm{i}}} \rho \mathrm{d} \mathbf{x} .
$$

\section{A paradigm problem in three dimensions}

We use the example of perfectly dielectric inclusions described in Section 3.4 to demonstrate the method developed in Section 2 in higher dimensions. We suppose that $\varepsilon$ and $\rho$ are periodic with period $\epsilon$, and that

$$
\begin{array}{ll}
\varepsilon=\varepsilon_{\mathrm{i}}, & \text { in } \Omega_{i}, \\
\varepsilon=\varepsilon_{\mathrm{e}}, & \text { in } \Omega_{e},
\end{array}
$$

where $\varepsilon_{i}, \varepsilon_{e}$ are constant.

We first apply the standard multiple-scales technique to the problem (3.1)-(3.3) with finite $\varepsilon_{\mathrm{i}}$, before taking the limit $\varepsilon_{\mathrm{i}} \rightarrow \infty$. We then apply multiple scales to the limiting problem (3.4)-(3.7), and show that the limits commute providing the integral constraint (3.7) is handled correctly.

\subsection{Standard multiple scales}

As usual, we write $\phi=\phi(\mathbf{x}, \mathbf{X})$ and suppose that $\phi$ is periodic in $\mathbf{X}$ with unit period, treating $\mathbf{x}$ and $\mathbf{X}$ as independent. The equation then becomes

$$
\left(\nabla_{\mathbf{x}}+\frac{1}{\epsilon} \nabla_{\mathbf{X}}\right) \cdot\left(\varepsilon(\mathbf{X})\left(\nabla_{\mathbf{x}}+\frac{1}{\epsilon} \nabla_{\mathbf{X}}\right)\right) \phi=-\rho(\mathbf{X}),
$$


with boundary conditions

$$
\begin{array}{r}
{[\phi]_{\mathrm{i}}^{\mathrm{e}}=0,} \\
{\left[\varepsilon \mathbf{n} \cdot\left(\nabla_{\mathbf{x}}+\frac{1}{\epsilon} \nabla_{\mathbf{X}}\right) \phi\right]_{\mathrm{i}}^{\mathrm{e}}=0,}
\end{array}
$$

Expanding $\phi=\phi_{0}+\epsilon \phi_{1}+\cdots$ gives at leading order

$$
\nabla_{\mathbf{X}}^{2} \phi_{0}=0 \quad \text { in } \Omega \equiv \Omega_{i} \cup \Omega_{e}
$$

with boundary conditions

$$
\begin{aligned}
{\left[\phi_{0}\right]_{\mathrm{i}}^{\mathrm{e}} } & =0, \\
{\left[\varepsilon \mathbf{n} \cdot \nabla_{\mathbf{X}} \phi_{0}\right]_{\mathrm{i}}^{\mathrm{e}} } & =0,
\end{aligned}
$$

and $\phi_{0}$ 1-periodic in $\mathbf{X}$. The only solution is for $\phi_{0}$ to be constant in $\mathbf{X}$, so that $\phi_{0}=\phi_{0}(\mathbf{x})$.

At next order

$$
\nabla_{\mathbf{X}}^{2} \phi_{1}=0 \quad \text { in } \Omega
$$

with boundary conditions

$$
\begin{aligned}
{\left[\phi_{1}\right]_{\mathrm{i}}^{\mathrm{e}} } & =0, \\
{\left[\varepsilon \mathbf{n} \cdot \nabla_{\mathbf{X}} \phi_{1}\right]_{\mathrm{i}}^{\mathrm{e}} } & =-[\varepsilon]_{\mathrm{i}}^{\mathrm{e}} \mathbf{n} \cdot \nabla_{\mathbf{x}} \phi_{0},
\end{aligned}
$$

and $\phi_{1} 1$-periodic in $\mathbf{X}$. The solution is

$$
\phi_{1}=\boldsymbol{\Psi} \cdot \nabla_{\mathbf{x}} \phi_{0}+\bar{\phi}_{1}(\mathbf{x})
$$

where $\boldsymbol{\Psi}=\left(\Psi_{1}, \Psi_{2}, \Psi_{3}\right)$ satisfies the cell problem

$$
\nabla_{\mathbf{X}}^{2} \Psi_{j}=0 \quad \text { in } \Omega
$$

with boundary conditions

$$
\begin{aligned}
{\left[\Psi_{j}\right]_{\mathrm{i}}^{\mathrm{e}} } & =0, \\
{\left[\varepsilon \mathbf{n} \cdot \nabla_{\mathbf{X}} \Psi_{j}\right]_{\mathrm{i}}^{\mathrm{e}} } & =-[\varepsilon]_{\mathrm{i}}^{\mathrm{e}} \mathbf{n} \cdot \mathbf{e}_{j},
\end{aligned}
$$

with $\mathbf{e}_{j}$ the unit vector in the $j$-direction, along with

$$
\int_{\Omega} \Psi_{j} \mathrm{~d} \mathbf{X}=\mathbf{0}
$$

At next order

$$
\begin{aligned}
\varepsilon_{i} \nabla_{\mathbf{X}} \cdot\left(\nabla_{\mathbf{X}} \phi_{2}+\nabla_{\mathbf{X}} \phi_{1}\right)+\varepsilon_{i} \nabla_{\mathbf{X}} \cdot\left(\nabla_{\mathbf{X}} \phi_{1}+\nabla_{\mathbf{x}} \phi_{0}\right)=-\rho(\mathbf{X}) & \text { in } \Omega_{i} \\
\varepsilon_{e} \nabla_{\mathbf{X}} \cdot\left(\nabla_{\mathbf{X}} \phi_{2}+\nabla_{\mathbf{x}} \phi_{1}\right)+\varepsilon_{e} \nabla_{\mathbf{X}} \cdot\left(\nabla_{\mathbf{X}} \phi_{1}+\nabla_{\mathbf{x}} \phi_{0}\right)=-\rho(\mathbf{X}) & \text { in } \Omega_{e}
\end{aligned}
$$


with boundary conditions

$$
\begin{aligned}
{\left[\phi_{2}\right]_{\mathrm{i}}^{\mathrm{e}} } & =0, \\
{\left[\varepsilon \mathbf{n} \cdot \nabla_{\mathbf{X}} \phi_{2}\right]_{\mathrm{i}}^{\mathrm{e}} } & =-\left[\varepsilon \mathbf{n} \cdot \nabla_{\mathbf{x}} \phi_{1}\right]_{\mathrm{i}}^{\mathrm{e}} .
\end{aligned}
$$

and $\phi_{2}$ 1-periodic in $\mathbf{X}$. Integrating over the unit cell $\Omega$ gives

$$
\nabla_{\mathbf{x}} \cdot\left(\varepsilon \nabla_{\mathbf{x}} \phi\right)=-\rho_{\mathrm{eff}},
$$

where

$$
\rho_{\text {eff }}=\int_{\Omega} \rho(\mathbf{X}) \mathrm{d} \mathbf{X},
$$

and the components of the effective conductivity tensor $\varepsilon$ are

$$
\varepsilon_{i j}=\int_{\Omega} \varepsilon\left(\delta_{i j}+\frac{\partial \Psi_{j}}{\partial X_{i}}\right) \mathrm{d} \mathbf{X} .
$$

\subsection{The limit $\varepsilon_{\mathrm{i}} \rightarrow \infty$}

If we let $\varepsilon_{\mathrm{i}} \rightarrow \infty$ in the cell problem (4.3)-(4.5), we find

$$
\nabla_{\mathbf{X}}^{2} \Psi_{j}=0 \quad \text { in } \Omega,
$$

with boundary conditions

$$
\begin{aligned}
{\left[\Psi_{j}\right]_{\mathrm{i}}^{\mathrm{e}} } & =0, \\
\mathbf{n} \cdot \nabla_{\mathbf{X}} \Psi_{j}^{\mathrm{i}} & =-\mathbf{n} \cdot \mathbf{e}_{j},
\end{aligned}
$$

which implies

$$
\boldsymbol{\Psi}=-\mathbf{X}+\text { constant } \quad \text { in } \Omega_{i} .
$$

The constant must be chosen so that (4.6) is satisfied.

We must also manipulate the expression for the conductivity tensor (4.7) which gives zero times infinity in the inclusion. We have

$$
\begin{aligned}
\varepsilon_{i j} & =\int_{\Omega} \varepsilon\left(\delta_{i j}+\frac{\partial \Psi_{j}}{\partial X_{i}}\right) \mathrm{d} \mathbf{X} \\
& =\int_{\Omega_{\mathrm{i}}} \varepsilon_{\mathrm{i}} \delta_{i j} \mathrm{~d} \mathbf{X}+\int_{\partial \Omega_{\mathrm{i}}} \varepsilon_{\mathrm{i}} X_{i} \frac{\partial \Psi_{j}}{\partial n} \mathrm{~d} S+\int_{\Omega_{\mathrm{e}}} \varepsilon_{\mathrm{e}} \delta_{i j} \mathrm{~d} \mathbf{X}-\int_{\partial \Omega_{\mathrm{i}}} \varepsilon_{\mathrm{e}} X_{i} \frac{\partial \Psi_{j}}{\partial n} \mathrm{~d} S+\int_{\partial \Omega} \varepsilon_{\mathrm{e}} X_{i} \frac{\partial \Psi_{j}}{\partial n} \mathrm{~d} S \\
& =\varepsilon_{\mathrm{i}}\left|\Omega_{\mathrm{i}}\right| \delta_{i j}+\varepsilon_{\mathrm{e}}\left|\Omega_{\mathrm{e}}\right| \delta_{i j}+[\varepsilon]_{\mathrm{i}}^{\mathrm{e}} \int_{\partial \Omega_{\mathrm{i}}} X_{i} n_{j} \mathrm{~d} S+\int_{\partial \Omega} \varepsilon_{\mathrm{e}} X_{i} \frac{\partial \Psi_{j}}{\partial n} \mathrm{~d} S \\
& =\varepsilon_{\mathrm{i}}\left|\Omega_{\mathrm{i}}\right| \delta_{i j}+\varepsilon_{\mathrm{e}}\left|\Omega_{\mathrm{e}}\right| \delta_{i j}+[\varepsilon]_{\mathrm{i}}^{\mathrm{e}} \int_{\Omega_{\mathrm{i}}} \delta_{i j} \mathrm{~d} S+\int_{\partial \Omega} \varepsilon_{\mathrm{e}} X_{i} \frac{\partial \Psi_{j}}{\partial n} \mathrm{~d} S \\
& =\varepsilon_{\mathrm{e}}|\Omega| \delta_{i j}+\int_{\partial \Omega} \varepsilon_{\mathrm{e}} X_{i} \frac{\partial \Psi_{j}}{\partial n} \mathrm{~d} S
\end{aligned}
$$

We can now safely take the limit $\varepsilon_{\mathrm{i}} \rightarrow \infty$. 


\subsection{Direct multiple scales}

We now apply the method of multiple scales directly to the limit problem (3.4)-(3.7). We first remark that, as in our one-dimensional example, it is crucial to write (3.5) as $\nabla \phi=0$ and not as $\phi=$ constant.

As usual, we write $\phi=\phi(\mathbf{x}, \mathbf{X})$ and suppose that $\phi$ is periodic in $\mathbf{X}$ with unit period, treating $\mathbf{x}$ and $\mathbf{X}$ as independent. Then

$$
\begin{aligned}
\varepsilon_{\mathrm{e}}\left(\nabla_{\mathbf{x}}+\frac{1}{\epsilon} \nabla_{\mathbf{X}}\right)^{2} \phi & =-\rho(\mathbf{X}) \quad \text { in } \Omega_{\mathrm{e}}, \\
\left(\nabla_{\mathbf{x}}+\frac{1}{\epsilon} \nabla_{\mathbf{X}}\right) \phi & =0 \quad \text { in } \Omega_{\mathrm{i}},
\end{aligned}
$$

with boundary condition

$$
[\phi]_{\mathrm{i}}^{\mathrm{e}}=0 .
$$

Expanding $\phi=\phi_{0}+\epsilon \phi_{1}+\cdots$ gives at leading order

$$
\begin{array}{ll}
\nabla_{\mathbf{X}}^{2} \phi_{0}=0 & \text { in } \Omega_{\mathrm{e}}, \\
\nabla_{\mathbf{X}} \phi_{0}=0 & \text { in } \Omega_{\mathrm{i}},
\end{array}
$$

with boundary condition

$$
\left[\phi_{0}\right]_{\mathrm{i}}^{\mathrm{e}}=0
$$

and $\phi_{0}$ 1-periodic in $\mathbf{X}$. The only solution is for $\phi_{0}$ to be constant in $\mathbf{X}$, so that $\phi_{0}=\phi_{0}(\mathbf{x})$.

At next order

$$
\begin{aligned}
\nabla_{\mathbf{X}}^{2} \phi_{1}=0 & \text { in } \Omega_{\mathrm{e}}, \\
\nabla_{\mathbf{X}} \phi_{1}+\nabla_{\mathbf{x}} \phi_{0}=0 & \text { in } \Omega_{\mathrm{i}},
\end{aligned}
$$

with boundary condition

$$
\left[\phi_{1}\right]_{\mathrm{i}}^{\mathrm{e}}=0
$$

and $\phi_{1} 1$-periodic in $\mathbf{X}$. The solution is

$$
\phi_{1}=\boldsymbol{\Psi} \cdot \nabla_{\mathbf{x}} \phi_{0}+\bar{\phi}_{1}(\mathbf{x})
$$

where $\boldsymbol{\Psi}=\left(\Psi_{1}, \Psi_{2}, \Psi_{3}\right)$ satisfies the cell problem

$$
\begin{aligned}
\nabla_{\mathbf{X}}^{2} \Psi_{j}=0 & \text { in } \Omega_{\mathrm{e}}, \\
\nabla_{\mathbf{X}} \Psi_{j}+\mathbf{e}_{j}=0 & \text { in } \Omega_{\mathrm{i}},
\end{aligned}
$$

with boundary condition

$$
\left[\Psi_{j}\right]_{\mathrm{i}}^{\mathrm{e}}=0
$$


with $\Psi_{j}$ 1-periodic in $\mathbf{X}$, where $\mathbf{e}_{j}$ the unit vector in the $j$-direction, along with

$$
\int_{\Omega} \Psi_{j} \mathrm{~d} \mathbf{X}=\mathbf{0}
$$

At next order

$$
\begin{aligned}
\varepsilon_{e} \nabla_{\mathbf{X}} \cdot\left(\nabla_{\mathbf{X}} \phi_{2}+\nabla_{\mathbf{x}} \phi_{1}\right)+\varepsilon_{e} \nabla_{\mathbf{x}} \cdot\left(\nabla_{\mathbf{X}} \phi_{1}+\nabla_{\mathbf{x}} \phi_{0}\right) & =-\rho(\mathbf{X}) \quad \text { in } \Omega_{\mathrm{e}} \\
\nabla_{\mathbf{X}} \phi_{2}+\nabla_{\mathbf{x}} \phi_{1} & =0 \quad \text { in } \Omega_{\mathrm{i}},
\end{aligned}
$$

with boundary condition

$$
\left[\phi_{2}\right]_{\mathrm{i}}^{\mathrm{e}}=0
$$

and $\phi_{2}$ 1-periodic in $\mathbf{X}$. Integrating (4.17) over $\Omega_{\mathrm{e}}$ gives

$$
\begin{aligned}
-\int_{\Omega_{\mathrm{e}}} \rho \mathrm{d} \mathbf{X}= & -\varepsilon_{\mathrm{e}} \int_{\partial \Omega_{\mathrm{i}}}\left(\nabla_{\mathbf{X}} \phi_{2}+\nabla_{\mathbf{x}} \phi_{1}\right) \cdot \mathbf{n} \mathrm{d} S+\varepsilon_{\mathrm{e}} \int_{\Omega_{\mathrm{e}}}\left(\nabla_{\mathbf{x}} \cdot \nabla_{\mathbf{X}} \phi_{1}+\nabla_{\mathbf{x}}^{2} \phi_{0}\right) \mathrm{d} \mathbf{X} \\
= & -\varepsilon_{\mathrm{e}} \int_{\partial \Omega_{\mathrm{i}}}\left(\nabla_{\mathbf{X}} \phi_{2}+\nabla_{\mathbf{x}} \phi_{1}\right) \cdot \mathbf{n} \mathrm{d} S+\varepsilon_{\mathrm{e}} \frac{\partial}{\partial x_{j}}\left(\left(\int_{\Omega_{\mathrm{e}}} \delta_{i j}+\frac{\partial \Psi_{i}}{\partial X_{j}} \mathrm{~d} \mathbf{X}\right) \frac{\partial \phi_{0}}{\partial x_{i}}\right) \\
= & -\varepsilon_{\mathrm{e}} \int_{\partial \Omega_{\mathrm{i}}}\left(\nabla_{\mathbf{X}} \phi_{2}+\nabla_{\mathbf{x}} \phi_{1}\right) \cdot \mathbf{n} \mathrm{d} S \\
& +\varepsilon_{\mathrm{e}} \frac{\partial}{\partial x_{j}}\left(\left(\delta_{i j}\left|\Omega_{\mathrm{e}}\right|-\int_{\partial \Omega_{\mathrm{i}}} X_{j} \frac{\partial \Psi_{i}}{\partial n} \mathrm{~d} S+\int_{\partial \Omega} X_{j} \frac{\partial \Psi_{i}}{\partial n} \mathrm{~d} S\right) \frac{\partial \phi_{0}}{\partial x_{i}}\right) .
\end{aligned}
$$

To evaluate the first integral on the right-hand side, we need to use the integral condition (3.7). As we saw in Section 2.1, we must take care when placing this condition in the multiple-scales framework.

\subsection{Dealing with the integral}

As before, it seems natural to write

$$
\int_{\partial \Omega_{\mathrm{i}}} \frac{\partial \phi}{\partial n} \mathrm{~d} S=\int_{\partial \Omega_{\mathrm{i}}}\left(\frac{1}{\epsilon} \nabla_{\mathbf{X}} \phi+\nabla_{\mathbf{x}} \phi\right) \cdot \mathbf{n} \mathrm{d} S,
$$

where $\partial \Omega_{\mathrm{i}}$ is the outer boundary of the inclusion in the unit cell $[-1 / 2,1 / 2]^{3}$. However, this is incorrect, as it neglects the small variation in $\mathbf{x}$ around $\partial \Omega_{\mathrm{i}}$.

Given an arbitrary point $\hat{\mathbf{x}}$, the interface is given by

$$
\left\{\mathbf{x}: \mathbf{x}=\hat{\mathbf{x}}-\epsilon \mathbf{b}+\epsilon \mathbf{X} \text { where } \mathbf{X} \in \partial \Omega_{\mathrm{i}}\right\},
$$

where $\mathbf{b}=\hat{\mathbf{x}} / \epsilon-\lfloor\hat{\mathbf{x}} / \epsilon\rfloor$. For ease of notation, we let $\mathbf{Q}=\varepsilon_{\mathrm{e}} \nabla \phi$. Then

$$
\begin{aligned}
\int_{\partial \Omega_{\mathrm{i}}} \mathbf{Q} \cdot \mathbf{n} \mathrm{d} S & =\epsilon^{2} \int_{\partial \Omega_{\mathrm{i}}} \mathbf{Q}(\hat{\mathbf{x}}-\epsilon \mathbf{b}+\epsilon \mathbf{X}, \mathbf{X}) \cdot \mathbf{n} \mathrm{d} S \\
& =\epsilon^{2} \int_{\partial \Omega_{\mathrm{i}}} \mathbf{Q}(\hat{\mathbf{x}}, \mathbf{X}) \cdot \mathbf{n} \mathrm{d} S+\epsilon^{3} \int_{\partial \Omega_{\mathrm{i}}}(\mathbf{b}+\mathbf{X}) \cdot \nabla_{\hat{\mathbf{x}}} \mathbf{Q}(\hat{\mathbf{x}}, \mathbf{X}) \cdot \mathbf{n} \mathrm{d} S+O\left(\epsilon^{4}\right) .
\end{aligned}
$$


Expanding $\mathbf{Q}=\mathbf{Q}_{0}+\epsilon \mathbf{Q}_{1}+\cdots$ we have

$$
\mathbf{Q}_{0}=\varepsilon_{\mathrm{e}}\left(\nabla_{\mathbf{X}} \phi_{1}+\nabla_{\mathbf{x}} \phi_{0}\right), \quad \mathbf{Q}_{1}=\varepsilon_{\mathrm{e}}\left(\nabla_{\mathbf{X}} \phi_{2}+\nabla_{\mathbf{x}} \phi_{1}\right) .
$$

Using (4.21) in (3.7) gives, at $O\left(\epsilon^{2}\right)$,

$$
\int_{\partial \Omega_{\mathrm{i}}} \mathbf{Q}_{0}(\hat{\mathbf{x}}, \mathbf{X}) \cdot \mathbf{n} \mathrm{d} S=0
$$

which is consistent with (4.12), and at $O\left(\epsilon^{3}\right)$,

$$
\begin{array}{r}
\int_{\partial \Omega_{\mathrm{i}}} \mathbf{Q}_{1}(\hat{\mathbf{x}}, \mathbf{X}) \cdot \mathbf{n} \mathrm{d} S+\int_{\partial \Omega_{\mathrm{i}}} \mathbf{X} \cdot \nabla_{\hat{\mathbf{x}}} \mathbf{Q}_{0}(\hat{\mathbf{x}}, \mathbf{X}) \cdot \mathbf{n} \mathrm{d} S \\
+\int_{\partial \Omega_{\mathrm{i}}} \mathbf{b} \cdot \nabla_{\hat{\mathbf{x}}} \mathbf{Q}_{0}(\hat{\mathbf{x}}, \mathbf{X}) \cdot \mathbf{n} \mathrm{d} S=-\int_{\Omega_{\mathrm{i}}} \rho \mathrm{d} \mathbf{X}
\end{array}
$$

However, from (4.23)

$$
\int_{\partial \Omega_{\mathrm{i}}} \mathbf{b} \cdot \nabla_{\hat{\mathbf{x}}} \mathbf{Q}_{0}(\hat{\mathbf{x}}, \mathbf{X}) \cdot \mathbf{n} \mathrm{d} S=0
$$

while

$$
\begin{aligned}
\int_{\partial \Omega_{\mathrm{i}}} \mathbf{X} \cdot \nabla_{\mathbf{x}}\left(\nabla_{\mathbf{x}} \phi_{0}\right) \cdot \mathbf{n} \mathrm{d} S & =\int_{\Omega_{\mathrm{i}}} \nabla_{\mathbf{X}} \cdot\left(\mathbf{X} \cdot \nabla_{\mathbf{x}}\left(\nabla_{\mathbf{x}} \phi_{0}\right)\right) \mathrm{d} \mathbf{X} \\
& =\int_{\Omega_{\mathrm{i}}} \nabla_{\mathbf{x}}^{2} \phi_{0} \mathrm{~d} \mathbf{X}=\left|\Omega_{\mathrm{i}}\right| \nabla_{\mathbf{x}}^{2} \phi_{0}
\end{aligned}
$$

Also,

$$
\begin{aligned}
\int_{\partial \Omega_{\mathrm{i}}}\left(\mathbf{X} \cdot \nabla_{\mathbf{X}}\left(\nabla_{\mathbf{X}} \phi_{1}\right)\right) \cdot \mathbf{n} \mathrm{d} S & =\int_{\partial \Omega_{\mathrm{i}}} X_{j} \frac{\partial}{\partial x_{j}} \frac{\partial \phi_{1}}{\partial X_{k}} n_{k} \mathrm{~d} S \\
& =\frac{\partial}{\partial x_{j}} \int_{\partial \Omega_{\mathrm{i}}} X_{j} \frac{\partial \Psi_{i}}{\partial X_{k}} \frac{\partial \phi_{0}}{\partial x_{i}} n_{k} \mathrm{~d} S \\
& =\frac{\partial}{\partial x_{j}}\left(\left(\int_{\partial \Omega_{\mathrm{i}}} X_{j} \frac{\partial \Psi_{i}}{\partial n} \mathrm{~d} S\right) \frac{\partial \phi_{0}}{\partial x_{i}}\right) .
\end{aligned}
$$

Thus

$$
\int_{\partial \Omega_{\mathrm{i}}} \mathbf{Q}_{1}(\hat{\mathbf{x}}, \mathbf{X}) \cdot \mathbf{n} \mathrm{d} S=-\int_{\Omega_{\mathrm{i}}} \rho \mathrm{d} \mathbf{X}-\varepsilon_{\mathrm{e}}\left|\Omega_{\mathrm{i}}\right| \nabla_{\mathbf{x}}^{2} \phi_{0}-\varepsilon_{\mathrm{e}} \frac{\partial}{\partial x_{j}}\left(\left(\int_{\partial \Omega_{\mathrm{i}}} X_{j} \frac{\partial \Psi_{i}}{\partial n} \mathrm{~d} S\right) \frac{\partial \phi_{0}}{\partial x_{i}}\right) .
$$

Returning to (4.19) and using (4.22) and (4.27)

$$
\begin{aligned}
-\int_{\Omega_{\mathrm{e}}} \rho \mathrm{d} \mathbf{X}= & \int_{\Omega_{\mathrm{i}}} \rho \mathrm{d} \mathbf{X}+\varepsilon_{\mathrm{e}}\left|\Omega_{\mathrm{i}}\right| \nabla_{\mathbf{x}}^{2} \phi_{0}+\varepsilon_{\mathrm{e}} \frac{\partial}{\partial x_{j}}\left(\left(\int_{\partial \Omega_{\mathrm{i}}} X_{j} \frac{\partial \Psi_{i}}{\partial n} \mathrm{~d} S\right) \frac{\partial p_{0}}{\partial x_{i}}\right) \\
& ++\varepsilon_{\mathrm{e}} \frac{\partial}{\partial x_{j}}\left(\left(\delta_{i j}\left|\Omega_{\mathrm{e}}\right|-\int_{\partial \Omega_{\mathrm{i}}} X_{j} \frac{\partial \Psi_{i}}{\partial n} \mathrm{~d} S+\int_{\partial \Omega} X_{j} \frac{\partial \Psi_{i}}{\partial n} \mathrm{~d} S\right) \frac{\partial p_{0}}{\partial x_{i}}\right) .
\end{aligned}
$$

Simplifying gives

$$
\nabla_{\mathbf{x}} \cdot\left(\varepsilon \nabla_{\mathbf{x}} \phi\right)=-\rho_{\mathrm{eff}}
$$


where

$$
\rho_{\mathrm{eff}}=\int_{\Omega} \rho(\mathbf{X}) \mathrm{d} \mathbf{X}
$$

and the effective conductivity tensor is

$$
\varepsilon_{i j}=\varepsilon_{\mathrm{e}}|\Omega| \delta_{i j}+\varepsilon_{\mathrm{e}} \int_{\partial \Omega} X_{i} \frac{\partial \Psi_{j}}{\partial n} \mathrm{~d} S,
$$

in agreement with (4.11).

\section{Acoustic waves in a bubbly fluid}

Let us finally return to the problem of acoustic waves in a bubbly fluid, to show that, contrary to the claim in [2], the multiple scales limit commutes with the limit of small gas density.

The equations for linearised motion about a periodic array of bubbles with centres $(i, j)$ are

$$
\begin{gathered}
\frac{\zeta^{*}}{C^{2}} \frac{\partial p}{\partial t}+\nabla \cdot \mathbf{u}=0, \\
\frac{\partial \mathbf{u}}{\partial t}+\zeta^{*} \nabla p=0,
\end{gathered}
$$

in the liquid region $\mathbf{x}:\left|\mathbf{x}-\mathbf{x}_{j}\right|>\delta$, and

$$
\begin{aligned}
& \frac{1}{\gamma} \frac{\partial p}{\partial t}+\nabla \cdot \mathbf{u}=0 \\
& \tau \frac{\partial \mathbf{u}}{\partial t}+\zeta^{*} \nabla p=0
\end{aligned}
$$

in the gas. At the interfaces $p$ and $\mathbf{u} \cdot \mathbf{n}$ are continuous. Here length, time, pressure and velocity have been nondimensionalised with $\lambda, 1 / f, p_{0}$ and $\bar{c}$ respectively, where $\lambda$ and $f$ are the wavelength and frequency of the propagating wave, $p_{0}$ is the pressure in the undisturbed fluid, $\bar{c}=\lambda f$ is the effective wavespeed, $C=c_{l} / \bar{c}$ where $c_{l}$ is the wavespeed of the liquid, $\delta$ is the nondimensional bubble radius, $\zeta^{*}=p_{0} / \rho_{l} \bar{c}^{2}, \tau=\rho_{g} / \rho_{l}$, and $\gamma$ is the ratio of specific heats in the gas, and $\rho_{g}$ and $\rho_{l}$ are the undisturbed densities of the gas and liquid respectively ${ }^{1}$

If we eliminate $\mathbf{u}$ as in [2] we find

$$
\frac{1}{C^{2}} \frac{\partial^{2} p}{\partial t^{2}}=\nabla^{2} p
$$

in the liquid region $\mathbf{x}:\left|\mathbf{x}-\mathbf{x}_{j}\right|>\delta$, and

$$
\frac{1}{\zeta^{*} \gamma} \frac{\partial^{2} p}{\partial t^{2}}=\frac{1}{\tau} \nabla^{2} p
$$

with $p$ continuous and $\left.\mathbf{n} \cdot \nabla p\right|_{l}=\left.\tau^{-1} \mathbf{n} \cdot \nabla p\right|_{g}$ on the interface.

1 Note that in [2] velocity is nondimensionalised with $\delta^{2} \bar{c}$, which leads to leading-order nondimensional velocities of $O\left(\delta^{-2}\right)$. However, in [2] $\mathbf{u}$ is eliminated to that velocity is not considered further. 
In [2] a multiple-scales analysis is performed and effective equations derived. They find that the leading-order pressure satisfies

$$
\sum_{i, j=1}^{3} q_{i j} \frac{\partial^{2} p}{\partial x_{i} \partial x_{j}}=\left(\frac{\left|\Omega_{B}\right|}{\gamma \zeta^{*}}+\frac{\left|\Omega_{F}\right|}{C^{2}}\right) \frac{\partial^{2} p}{\partial t^{2}},
$$

where

$$
q_{i j}=\left\langle a\left(\frac{\partial \chi_{i}}{\partial X_{j}}+\delta_{i j}\right)\right\rangle,
$$

and $\chi_{i}=\chi_{i}(\mathbf{X})$ are solutions of the cell problems

$$
\nabla_{\mathbf{X}} \cdot\left(a(\mathbf{X})\left(\nabla_{\mathbf{X}} \chi_{i}+\mathbf{e}_{i}\right)\right)=0 \quad \text { in } \Omega
$$

where

$$
a(\mathbf{X})= \begin{cases}\tau^{-1} & \text { if } \mathbf{X} \in \Omega_{B}, \\ 1 & \text { if } \mathbf{X} \in \Omega_{F},\end{cases}
$$

where $\Omega_{B}$ and $\Omega_{F}$ are the bubble and fluid regions of the unit cell $\Omega=\Omega_{B} \cup \Omega_{F}$ respectively. It is claimed in [2] that the multiple-scales limit $\epsilon \rightarrow 0$ is not interchangeable with the low gas-bubble-inertia limit $\tau \rightarrow 0$. Here we aim to show that this is not the case, that is, the limits are interchangeable, providing the analysis is performed carefully.

If we let $\tau \rightarrow 0$ in the cell problem (5.9), we find that $\chi_{i}$ satisfies

$$
\begin{array}{rl}
\nabla_{\mathbf{X}} \chi_{i}+\mathbf{e}_{i}=0 & \mathbf{X} \in \Omega_{B}, \\
\nabla_{\mathbf{X}}^{2} \chi_{i}=0 & \mathbf{X} \in \Omega_{F},
\end{array}
$$

with $\chi_{i}$ continuous across the bubble interface $\Gamma$, and 1-periodic in $\mathbf{X}$. However, it is not so straightforward to see the contribution to $q_{i j}$ in (5.8) from the bubble region (since it involves zero times infinity). Following the same procedure as in (4.11), we find

$$
\begin{aligned}
q_{i j} & =\left(\frac{\left|\Omega_{B}\right|}{\tau}+\left|\Omega_{F}\right|\right) \delta_{i j}+\frac{1}{\tau} \int_{\Omega_{B}} \frac{\partial \chi_{i}}{\partial X_{j}} \mathrm{~d} \mathbf{X}+\int_{\Omega_{F}} \frac{\partial \chi_{i}}{\partial X_{j}} \mathrm{~d} \mathbf{X} \\
& =\left(\frac{\left|\Omega_{B}\right|}{\tau}+\left|\Omega_{F}\right|\right) \delta_{i j}-\int_{\Gamma} X_{j}\left[a \frac{\partial \chi_{i}}{\partial n}\right]_{-}^{+} \mathrm{d} S+\int_{\partial \Omega} X_{j} \frac{\partial \chi_{i}}{\partial n} \mathrm{~d} S \\
& =\left(\frac{\left|\Omega_{B}\right|}{\tau}+\left|\Omega_{F}\right|\right) \delta_{i j}+\int_{\Gamma} X_{j}\left[a n_{i}\right]_{-}^{+} \mathrm{d} S+\int_{\partial \Omega} X_{j} \frac{\partial \chi_{i}}{\partial n} \mathrm{~d} S \\
& =\delta_{i j}+\int_{\partial \Omega} X_{j} \frac{\partial \chi_{i}}{\partial n} \mathrm{~d} S .
\end{aligned}
$$

\subsection{Integral constraint}

In the limit $\tau \rightarrow 0,(5.5),(5.6)$ become

$$
\frac{1}{C^{2}} \frac{\partial^{2} p}{\partial t^{2}}=\nabla^{2} p
$$


in the liquid region $\mathbf{x}:\left|\mathbf{x}-\mathbf{x}_{j}\right|>\delta$, and

$$
\nabla p=0
$$

with $p$ continuous. As in our paradigm problem, in this limit we have lost a boundary condition, and need to add an integral condition to complete the formulation. Integrating (5.6) over the bubble surface $\Gamma$ gives this condition as

$$
\left.\int_{\Gamma} \frac{\partial p}{\partial n}\right|_{F} \mathrm{~d} S=\int_{\Omega_{B}} \frac{1}{\zeta^{*} \gamma} \frac{\partial^{2} p}{\partial t^{2}} \mathrm{~d} V=\frac{\left|\Omega_{B}\right|}{\zeta^{*} \gamma} \frac{\partial^{2} p_{g}}{\partial t^{2}},
$$

since $p$ is uniform in the bubble by (5.15).

We write $\mathbf{x}=\epsilon \mathbf{X}$ and $p=p(\mathbf{x}, \mathbf{X}, t)$ and require that $p$ be 1-periodic in $\mathbf{X}$. Expanding

$$
p=p_{0}(\mathbf{x}, \mathbf{X}, t)+\epsilon p_{1}(\mathbf{x}, \mathbf{X}, t)+\cdots,
$$

the procedure is very similar to Section 3.3 and gives $p_{0}=p_{0}(\mathbf{x}, t)$ and

$$
p_{1}(\mathbf{x}, \mathbf{X}, t)=\sum_{i=1}^{3} \Psi_{i}(\mathbf{X}) \frac{\partial p_{0}}{\partial x_{i}}+\bar{p}_{1}(\mathbf{x}, t)=\Psi(\mathbf{X}) \cdot \nabla_{\mathbf{x}} p_{0}+\bar{p}_{1}(\mathbf{x}, t)
$$

where $\Psi$ satisfies the cell problem

$$
\begin{array}{rl}
\nabla_{\mathbf{X}}^{2} \Psi_{i}=0 & \mathbf{X} \in \Omega_{F}, \\
\nabla_{\mathbf{X}} \Psi_{i}+\mathbf{e}_{i}=0 & \mathbf{X} \in \Omega_{B},
\end{array}
$$

with $\Psi_{i}$ continuous and 1-periodic in $\mathbf{X}$, and

$$
\int_{\Omega} \Psi(\mathbf{X}) \mathrm{d} \mathbf{X}=\mathbf{0}
$$

Thus $\Psi_{i}$ is identical to the limiting $\chi_{i}$ of $(5.11),(5.12)$ as we would expect. We see that

$$
\Psi=-\mathbf{X}+\text { const. } \quad \mathbf{X} \in \Omega_{B} .
$$

This variation of $p_{1}$ with $\mathbf{X}$ inside the bubble was one of the reasons given in [2] for the non-commutativity of the limits: the multiple scales expansion appears to give a non-constant pressure in the bubble, while in the limit $\tau \rightarrow 0$ the pressure in the bubble must be constant. However, this variation in $p_{1}$ with $\mathbf{X}$ is to be expected, and is just what is needed to cancel the variation of $p_{0}$ with $\mathbf{x}$ to give a constant pressure in the bubble.

Equating coefficients at the next order in $\epsilon$ gives, finally,

$$
\begin{aligned}
\nabla_{\mathbf{X}}^{2} p_{2}+\nabla_{\mathbf{X}} \cdot\left(\nabla_{\mathbf{x}} p_{1}\right)+\nabla_{\mathbf{x}} \cdot\left(\nabla_{\mathbf{x}} p_{1}\right)+\nabla_{\mathbf{x}}^{2} p_{0} & =\frac{1}{C^{2}} \frac{\partial^{2} p_{0}}{\partial t^{2}} \quad \mathbf{X} \in \Omega_{F}, \\
\nabla_{\mathbf{X}} p_{2}+\nabla_{\mathbf{x}} p_{1} & =0 \quad \mathbf{X} \in \Omega_{B},
\end{aligned}
$$

with $p_{2}$ continuous and 1-periodic in $\mathbf{X}$. Integrating (5.20) over the fluid domain in the 
unit cell gives

$$
\int_{\partial \Omega_{F}}\left(\nabla_{\mathbf{X}} p_{2}+\nabla_{\mathbf{x}} p_{1}\right) \cdot \mathbf{n} \mathrm{d} S=\frac{\left|\Omega_{F}\right|}{C^{2}} \frac{\partial^{2} p_{0}}{\partial t^{2}}-\frac{\partial}{\partial x_{j}}\left(\left(\left|\Omega_{F}\right| \delta_{i j}+\int_{\partial \Omega_{F}} X_{j} \frac{\partial \Psi_{i}}{\partial n} \mathrm{~d} S\right) \frac{\partial p_{0}}{\partial x_{i}}\right) .
$$

For $\tau>0$ the left-hand side of (5.22) is evaluated through continuity of flux after a similar integral over the bubble. Here, however, we need to use the integral condition (5.16). Given an arbitrary point $\hat{\mathbf{x}}$ the bubble surface is given by

$$
\left\{\mathbf{x}: \mathbf{x}=\hat{\mathbf{x}}-\epsilon \mathbf{b}+\epsilon \mathbf{X} \text { where } \mathbf{X} \in \partial \Omega_{B}\right\},
$$

where $\mathbf{b}=\hat{\mathbf{x}} / \epsilon-\lfloor\hat{\mathbf{x}} / \epsilon\rfloor$. If we let $\mathbf{Q}=\nabla p$ then, as in Section 3.3, expanding $\mathbf{Q}=$ $\mathbf{Q}_{0}+\epsilon \mathbf{Q}_{1}+\cdots$ we have

$$
\mathbf{Q}_{0}=\nabla_{\mathbf{x}} p_{1}+\nabla_{\mathbf{x}} p_{0}, \quad \mathbf{Q}_{1}=\nabla_{\mathbf{x}} p_{2}+\nabla_{\mathbf{x}} p_{1}
$$

and

$$
\int_{\partial \Omega_{B}} \mathbf{Q}_{1}(\hat{\mathbf{x}}, \mathbf{X}) \cdot \mathbf{n} \mathrm{d} S(\mathbf{X})+\int_{\Omega_{B}} \mathbf{X} \cdot \nabla_{\hat{\mathbf{x}}} \mathbf{Q}_{0}(\hat{\mathbf{x}}, \mathbf{X}) \cdot \mathbf{n} \mathrm{d} S(\mathbf{X})=\frac{\left|\Omega_{B}\right|}{\zeta^{*} \gamma} \frac{\partial^{2} p_{0}}{\partial t^{2}}
$$

As in Section 3.3

$$
\int_{\partial \Omega_{B}} \mathbf{X} \cdot \nabla_{\mathbf{x}}\left(\nabla_{\mathbf{x}} p_{0}\right) \cdot \mathbf{n} \mathrm{d} S(\mathbf{X})=\int_{\Omega_{B}} \nabla_{\mathbf{x}}^{2} p_{0} \mathrm{~d} \mathbf{X}=\left|\Omega_{B}\right| \nabla_{\mathbf{x}}^{2} p_{0}
$$

Also,

$$
\begin{aligned}
\int_{\partial B^{+}}\left(\mathbf{X} \cdot \nabla_{\mathbf{X}}\left(\nabla_{\mathbf{X}} p_{1}\right)\right) \cdot \mathbf{n} \mathrm{d} S(\mathbf{X}) & =\int_{\partial B^{+}} X_{j} \frac{\partial}{\partial x_{j}} \frac{\partial p_{1}}{\partial X_{k}} n_{k} \mathrm{~d} S(\mathbf{X}) \\
& =\frac{\partial}{\partial x_{j}} \int_{\partial B^{+}} X_{j} \frac{\partial \Psi_{i}}{\partial X_{k}} \frac{\partial p_{0}}{\partial x_{i}} n_{k} \mathrm{~d} S(\mathbf{X}) \\
& =\frac{\partial}{\partial x_{j}}\left(\int_{\partial B^{+}} X_{j} \frac{\partial \Psi_{i}}{\partial n} \mathrm{~d} S(\mathbf{X}) \frac{\partial p_{0}}{\partial x_{i}}\right) .
\end{aligned}
$$

Using (5.23)-(5.26) in (5.22) gives

$$
\left(\frac{\left|\Omega_{F}\right|}{C^{2}}+\frac{|B|}{\zeta^{*} \gamma}\right) \frac{\partial^{2} p_{0}}{\partial t^{2}}=\frac{\partial}{\partial x_{j}}\left(\left(\delta_{i j}+\int_{\partial D} X_{j} \frac{\partial \Psi_{i}}{\partial n} \mathrm{~d} S(\mathbf{X})\right) \frac{\partial p_{0}}{\partial x_{i}}\right)
$$

as required.

\section{Conclusion}

We have shown how to handle multiple-scales homogenisation problems in which an integral constraint is applied over each inclusion. Care must be taken in determining the location of the integral surface in the multiple scales coordinates: the obvious formulation leads to incorrect results. 
It is natural to ask why the additional terms we find are not present in all multiplescales problems involving interfaces. For example, why are they not also present when the boundary conditions at the interface are continuity conditions. Although in principle in this case also care should be taken as to the exact location of the interface, the correction terms we find evaluate to zero in this case because of the continuity of the underlying function.

We illustrated the problem and the solution first with a one-dimensional paradigm. We then showed how to apply the method in three dimensions, using the problem of perfectly dielectric inclusions as an example. Finally, we applied our methods to show that the low-gas-density limit and the multiple-scales limit for the propagation of acoustic waves through a bubbly fluid commute, in contrast to previous claims.

Knowing how to handle correctly, conditions on the interface between fluid and bubble allows us to consider more complicated scenarios. For example, it makes it easier to consider the case of surface tension between the bubbles and the liquid, which is difficult to write in conservation form. The results are not straightforward, and are too involved to be included here; this will be the subject of a future paper.

\section{References}

[1] Bensoussan, A., Lions, J.-L. \& Papanicolaou, G. (1978) Asymptotic Analysis for Periodic Structures, Studies in Mathematics and Its Applications, Vol. 5, North-Holland Publishing Company, Amsterdam.

[2] Caflisch, R. E., Miksis, M. J., Papanicolaou, G. C. \& Ting, L. (1985) Wave propagation in bubbly liquids at finite volume fraction. J. Fluid. Mech. 160, 1-14.

[3] Davit, Y., Bell, C. G., Byrne, H. M., Chapman, L. A. C., Kimpton, L. S., Lang, G. E., Leonard, K. H. L., Oliver, J. M., Pearson, N. C., Shipley, R. J., Waters, S. L., Whiteley, J. P., WoOD, B. D. \& Quintard, M. (2013) Homogenization via formal multiscale asymptotics and volume averaging: How do the two techniques compare? Adv. Water Resour. 62, 178-206.

[4] Hashin, Z. \& Shtrikman, S. (1962) A variational approach to the theory of the effective magnetic permeability of multiphase materials. J. Appl. Phys. 33, 3125.

[5] Hinch, E. J. (1991) Perturbation Methods, Cambridge University Press.

[6] Kevorkian, J. K. \& Cole, J. D. (1981) Perturbation Methods in Applied Mathematics, SpringerVerlag, Berlin.

[7] Pavliotis, G. A. \& Stuart, A. M. (2008) Multiscale Methods: Averaging and Homogenization, Springer Science + Business Media, LLC.

[8] Torquato, S. (1991) Random heterogeneous media: Microstructure and improved bounds on effective properties. Appl. Mech. Rev. 44, 37.

[9] Torquato, S. (2000) Modeling of physical properties of composite materials. Int. J. Solids Struct. 37, 411-422.

[10] Whitaker, S. (1999) The Method of Volume Averaging, Theory and Applications of Transport in Porous Media, Vol. 13, Springer Science + Business Media, Dordrecht. 